\title{
On Shifted Weibull-Pareto Distribution
}

\author{
Ahmad Alzaghal ${ }^{1}$, Indranil Ghosh ${ }^{2} \&$ Ayman Alzaatreh $^{3}$ \\ ${ }^{1}$ Department of Mathematics, State University of New York at Oswego, Oswego, USA \\ ${ }^{2}$ Department of Mathematics and Statistics, University of North Carolina Wilmington, Wilmington, NC \\ ${ }^{3}$ Department of Mathematics, Nazarbayev University, Astana, KZ
}

Correspondence: Ahmad Alzaghal, Department of Mathematics, State University of New York at Oswego, Oswego, USA. Tel: 1-315-312-2728. E-mail: ahmad.alzaghal@oswego.edu.

Received: April 12, 2016 Accepted: April 24, 2016 Online Published: June 27, 2016

doi:10.5539/ijsp.v5n4p139

URL: http://dx.doi.org/10.5539/ijsp.v5n4p139

\begin{abstract}
The Lomax distribution, known as Pareto (type II) distribution, is a heavy tail probability distribution used extensively in business, economics and in actuarial modeling. The Weibull-Pareto distribution defined by Alzaatreh et al. (2013a) has shown high bias and standard error for the ML estimates when the parameter $c>>1$. In this paper we use the Lomax distribution to construct the Weibull-Lomax distribution. It is observed that the Weibull-Lomax distribution performs significantly better in terms of the ML estimations. Some structural properties of the Weibull-Lomax distribution are discussed.
\end{abstract}

Keywords: Lomax distribution, Weibull-Lomax distribution, $T-X$ family of distributions

\section{Introduction}

Let $r(t)$ be the probability density function (PDF) of a random variable $T \in[a, b]$ for $-\infty \leq a<b \leq \infty$ and let $G(x)$ be the cumulative distribution function (CDF) of a random variable $X$ such that the link function $W(\cdot):[0,1] \longrightarrow[a, b]$ satisfies the following conditions: (i) $W(\cdot)$ is differentiable and monotonically non-decreasing, and (ii) $W(0) \rightarrow a$ and $W(1) \rightarrow b$.

The CDF of the T-X family defined by Alzaatreh et al. (2013b) is

$$
F(x)=\int_{a}^{W(G(x))} r(t) d t .
$$

If the random variable $T \in(0, \infty)$ and $W(z)=-\log (1-z)$, then the PDF corresponding to (1) is given by

$$
f(x)=h_{g}(x) R\left(H_{g}(x)\right),
$$

where $h_{g}=g /(1-G)$ and $H_{g}=-\log (1-G)$ are the hazard and cumulative hazard rate functions corresponding to the PDF $g(x)$.

Alzaatreh et al. (2013a) studied a generalization of the Pareto distribution by using (2) where $T$ and $X$ follow Weibull and Pareto random variables respectively. The probability density function (PDF) of the Weibull-Pareto distribution (WPD) is defined as

$$
f(x)=\frac{\beta c}{x}\left(\beta \log \left(\frac{x}{\theta}\right)\right)^{c-1} \mathrm{e}^{-(\beta \log (x / \theta))^{c}}, \quad x>\theta ; c, \beta, \theta>0 .
$$

Alzaatreh et al. (2013a) studied some general properties of the Weibull-Pareto distribution in (3). They showed that this distribution can be applied to fit data with different characteristics. It can fit data with long right tail, long left tail and approximately symmetric. However, they pointed out a major problem in estimating the parameters of the Weibull-Pareto distribution (WPD). The estimated values of the parameters $c$ and $\beta$ using the maximum likelihood estimation (MLE) have high biases and standard errors when $c>>1$. This occurred because when $c>1$, the WPD can be left skewed. The left skeweness of the WPD affects the ML estimates of the parameters. It was shown that the estimates of $c$ and $\beta$ are very sensitive to the estimate of the parameter $\theta$. To solve this problem, Alzaatreh et al. (2013a) proposed a modification of the MLE method (MMLE) which improved the results of the MLE. However, they pointed out that MMLE still produce high bias and standard error values in some cases and further research needed to solve the estimation problem for the parameters of WPD. The main objective of this article is to provide an alternative to the Weibull-Pareto distribution namely, the Weibull-Lomax distribution. By merely considering another member of the family of Pareto distribution 
(after suitable transformation) a greater improvement in estimation under the MLE (maximum likelihood method) is achieved. The paper is organized as follows: In section 2, the Weibull-Lomax distribution is defined and several properties are studied including quantile function, limit behaviour, unimoadality, Shannon entropy, reliability parameter, moments, mean deviations and order statistics. The asymptotic distributions of the sample minima and maxima are also studied in section 2. Parameter estimation and application are studied in section 3. We provide some concluding remarks in section 4.

\section{The Weibull-Lomax Distribution}

Let the random variable $T$ and $X$ follow the Weibull and Lomax distributions. Then from (2), the PDF of the Weibulllomax distribution (WLD) can be written as

$$
f(x)=\frac{\beta c}{x+\theta}\left(\beta \log \left(1+\frac{x}{\theta}\right)\right)^{c-1} \mathrm{e}^{-\left(\beta \log \left(1+\frac{x}{\theta}\right)\right)^{c}}, \quad x>0 ; c, \beta, \theta>0 .
$$

Remark 2.1. Note that the WLD in (4) is only a shift by $\theta$ of the WPD in (3). I.e. If $Y \sim W P D(c, \beta, \theta)$ then $X=Y-\theta$ follows $W L D(c, \beta, \theta)$.

When $c=1$, the WLD reduces to the Lomax distribution with parameters $\beta$ and $\theta$. From (4), the CDF and hazard rate function of WLD, respectively, are

$$
\begin{gathered}
F(x)=1-\mathrm{e}^{-\left(\beta \log \left(1+\frac{x}{\theta}\right)\right)^{c}} . \\
h(x)=\frac{\beta c}{x+\theta}\left(\beta \log \left(1+\frac{x}{\theta}\right)\right)^{c-1},
\end{gathered}
$$

In Figures 1 and 2, various graphs of $f(x)$ and $h(x)$ are provided for different parameter values. The plots indicate that the Weibull-Lomax distribution can be reverse $J$-shaped, right-skewed or left-skewed. Also, the Weibull-Lomax distribution hazard function can be a decreasing failure rate or upside down bathtub shapes.

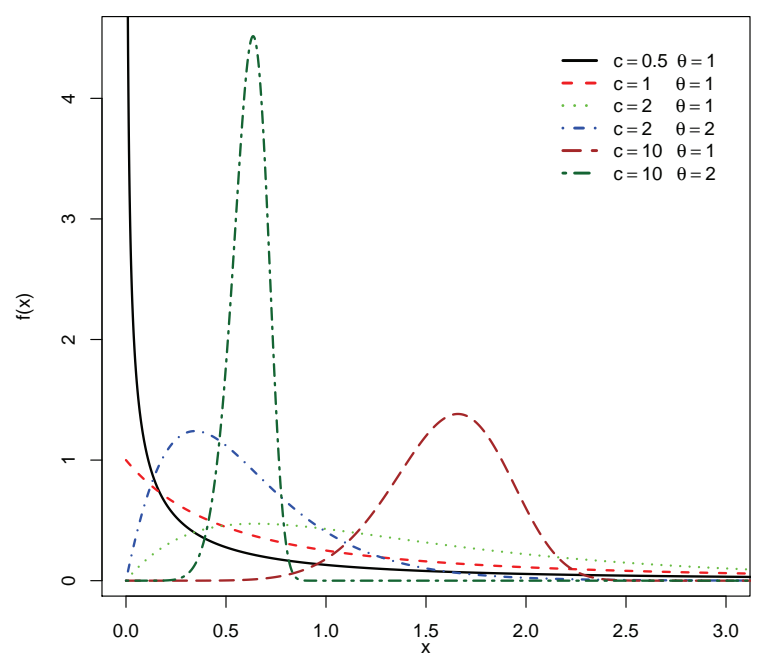

Figure 1. Graphs of the Weibull-Lomax PDF for various choices of $c$ and $\theta$ when $\beta=1$.

Remark 2.2. Based on Remark 2.1 and Alzaatreh et al. (2013a), one can obtain the following properties of the WLD.

(i) If a random variable $Y$ follows the Weibull distribution with parameters $c$ and $1 / \beta$, then the random variable $X=\theta\left(\mathrm{e}^{Y}-1\right)$ follows the Weibull-Lomax distribution.

(ii) Let $Q(p), 0<p<1$ denote the quantile function of WLD. Then $Q(p)=\theta\left\{\exp \left((-\log (1-p))^{1 / c} / \beta\right)-1\right\}$. 


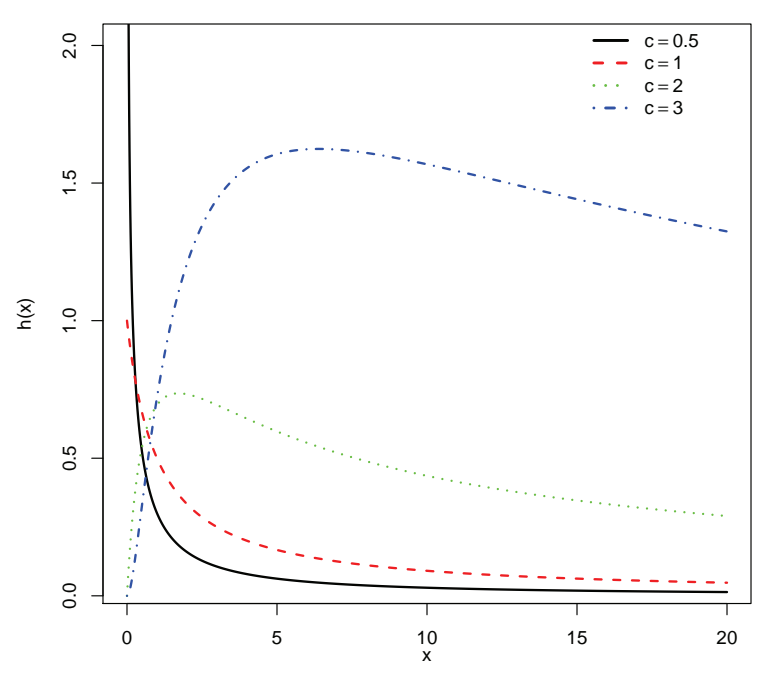

Figure 2. Graphs of the Weibull-Lomax hazard function for various choices of $c$ when $\beta=\theta=1$

(iii) The limit of the Weibull-Lomax density and the Weibull-Lomax hazard function as $x \rightarrow \infty$ is 0 , and the limit as $x \rightarrow 0$ is given by

$$
\lim _{x \rightarrow 0} f(x)=\lim _{x \rightarrow 0} h(x)= \begin{cases}0, & c>1 \\ \beta / \theta, & c=1 . \\ \infty, & c<1\end{cases}
$$

(iv) The WLD is unimodal at $x_{0}$. When $c \leq 1$, the mode is at $x_{0}=0$ and when $c>1$, the mode is the solution of the equation $k\left(x_{0}\right)=0$, where

$$
k(x)=-\log (1+x / \theta)-c(\beta \log (1+x / \theta))^{c}+c-1 .
$$

(v) The Shannon entropy (Shannon, 1948) for a random variable $X$ that follows the WLD is

$$
\eta_{X}=-\log \left(\frac{\beta c}{\theta}\right)+\frac{1}{\beta} \Gamma\left(1+\frac{1}{c}\right)+\left(1-\frac{1}{c}\right) \delta+1,
$$

where $\delta=-\int_{0}^{\infty} e^{-u} \log (u) d u=0.57722$ is the Euler gamma constant.

\subsection{Reliability Parameter}

The reliability parameter $R$ is defined as $R=P(X>Y)$, where $X$ and $Y$ are independent random variables. Numerous applications of the reliability parameter have appeared in the literature such as the area of classical stress-strength model and the break down of a system having two components. Other applications of the reliability parameter can be found in Hall (1984) and Weerahandi and Johnson (1992). If $X$ and $Y$ are two continuous and independent random variables with the CDFs $F_{1}(x)$ and $F_{2}(y)$ and their PDFs $f_{1}(x)$ and $f_{2}(y)$ respectively, then the reliability parameter $R$ can be written as

$$
R=P(X>Y)=\int_{-\infty}^{\infty} F_{2}(t) f_{1}(t) d t .
$$

Theorem 2.3. Suppose that $X \sim W L D\left(c_{1}, \beta_{1}, \theta\right)$ and $Y \sim W L D\left(c_{2}, \beta_{2}, \theta\right)$, then

$$
R=1-\sum_{k=0}^{\infty} \frac{(-1)^{k}}{k !}\left(\frac{\beta_{2}}{\beta_{1}}\right)^{k c_{2}} \Gamma\left(\frac{k c_{2}}{c_{1}}+1\right) .
$$

Proof. Follows from Lemma 2.4 in Alzaatreh and Ghosh (2014). 


\subsection{Moments and Mean Deviations}

Using Remark 2.1, the $s$-th moments for the WLD can be written as

$$
E\left(X^{s}\right)=(-1)^{s} \theta^{s}+\theta^{s} \sum_{k=1}^{s} \sum_{i=0}^{\infty}\left(\begin{array}{l}
s \\
k
\end{array}\right) \frac{(-1)^{s-k} k^{i}}{i ! \beta^{i}} \Gamma(1+i / c) .
$$

Remark 2.4. From (7), we have the following

(i) If $c>1$, then the $s$-th moments of the Weibull-Lomax distribution exist.

(ii) If $c<1$, then the $s$-th moments of the Weibull-Lomax distribution do not exist.

(iii) If $c=1$, then the $s$-th moments of the Weibull-Lomax distribution exist iff $\beta>s$.

The amount of scatter in a population is evidently measured to some extent by the totality of deviations from the mean and median. If we denote the median by $M$, then the mean deviation from the mean, $D(\mu)$, and the mean deviation from the median, $D(M)$, can be written as

$$
\begin{gathered}
D(\mu)=2 \mu F(\mu)-2 \int_{0}^{\mu} x f(x) d x . \\
D(M)=\mu-2 \int_{0}^{M} x f(x) d x .
\end{gathered}
$$

Now, consider $I_{m}=\int_{0}^{m} x f(x) d x$, where $f(x)$ is defined in (4). On Using the substitution $u=(\beta \log (1+x / \theta))^{c}$, we get

$$
\begin{aligned}
I_{m} & =\theta \sum_{k=0}^{\infty} \frac{1}{\beta^{k} k !} \int_{0}^{a_{m}} u^{k / c} \mathrm{e}^{-u} d u \\
& =\theta \sum_{k=0}^{\infty} \frac{\gamma\left(a_{m}, 1+k / c\right)}{\beta^{k} k !}, \quad c>1,
\end{aligned}
$$

where $m>0, a_{m}=(\beta \log (1+m / \theta))^{c}$ and $\gamma(x, a)=\int_{0}^{x} t^{a-1} e^{-t} d t$ is the lower incomplete gamma function.

From equations (8) and (9), the mean deviation from the mean and the mean deviation from the median are, respectively, given by

$$
\begin{aligned}
D(\mu) & =2 \mu\left(1-\exp \left\{-(\beta \log (1+\mu / \theta))^{c}\right\}\right)-2 I_{\mu} \\
D(M) & =\mu-2 I_{M},
\end{aligned}
$$

where $I_{\mu}$ and $I_{M}$ can be calculated numerically from (10).

\subsection{Quantile Measures of Tail Behavior}

Skewness and kurtosis of a distribution can be measured by $\beta_{1}=\mu_{3} / \sigma^{3}$ and $\beta_{2}=\mu_{4} / \sigma^{4}$, respectively. However the expression for the third and fourth moments of $\operatorname{WLD}(c, \beta, \theta)$ do not always exist (see Remark 2.4). Consequently, the moment based skewness and kurtosis measures can not be applied when $c<1$. Instead we consider the quantile based skewness and kurtosis measures which always exist. The quantile function of $\operatorname{WLD}(c, \beta, \theta)$ are in closed form, alternatively we can define the measure of skewness and kurtosis based on the quantile function. The Galton' skewness $S$ defined by Galton (1883) and the Moors' kurtosis $K$ defined by Moors (1988) are given by

$$
\begin{gathered}
S=\frac{Q(6 / 8)-2 Q(4 / 8)+Q(2 / 8)}{Q(6 / 8)-Q(2 / 8)} . \\
K=\frac{Q(7 / 8)-Q(5 / 8)+Q(3 / 8)-Q(1 / 8)}{Q(6 / 8)-Q(2 / 8)} .
\end{gathered}
$$

When the distribution is symmetric, $S=0$ and when the distribution is right (or left) skew, $S>0$ (or $S<0$ ). As $K$ increases the tail of the distribution becomes heavier. To investigate the effect of the two shape parameters $c$ and $\beta$ on the $\operatorname{WLD}(c, \beta, \theta)$ distribution, equation (11) and (12) are used to obtain the Galtons' skewness and Moors' kurtosis where 
the quantile function can be found from Remark 2.2. Figure 3 displays 3D graphs for the Galtons' skewness and Moors' kurtosis for the $\operatorname{WLD}(c, \beta, \theta)$ when $\theta=1$ and different values of $c$ and $\beta$. From Figure 3, the WLD can be left skewed, right skewed or near symmetric $(\mathrm{S}=0)$. Furthermore, for fixed value of $c>1$, Galton's skewness and Moors' kurtosis are decreasing function of $\beta$. Also, for fixed value of $\beta$, Galton's skewness and Moors' kurtosis are decreasing function of $c$. In Figure 4, we determined the regions when the WLD is left skewed, right skewed or nearly symmetric $(\mathrm{S}=0)$ occur. A cubic function relating $\log (\beta)$ to $\log (c)$ is obtained for the situation when the distribution is nearly symmetric (see $\mathrm{R}$ results in the Appendix). Since the symmetry of WLD does not depend on $\theta$, we assumed $\theta=1$.
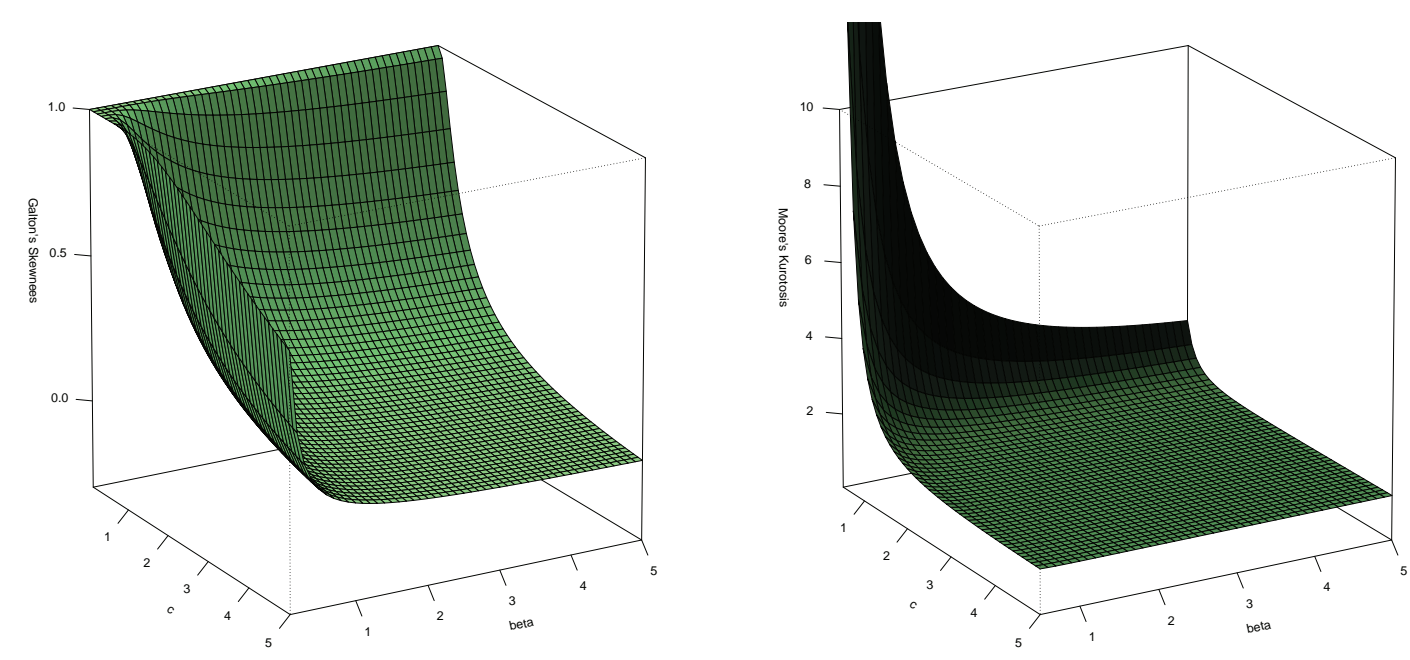

Figure 3. Graphs of Quantile Skewness and Kurtosis for the WL PDF when $\theta=1$.

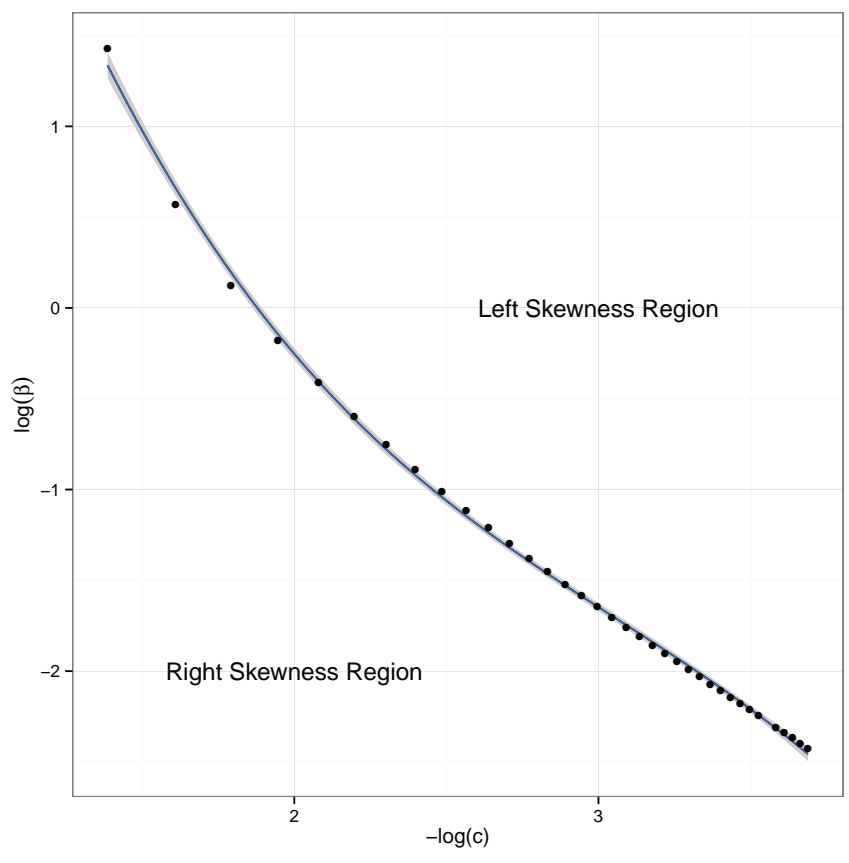

Figure 4. Skewness regions for the WLD.

\subsection{Order Statistics for the Weibull Lomax Distribution}

In this section, we study the moments of the $r$-th order statistic and the large sample distribution of the sample minimum and the sample maximum when a random sample of size $n$ are drawn from the $\operatorname{WLD}(c, \beta, \theta)$ distribution. The density 
function of the $r$-th order statistic $X_{r: n}$ for a random sample of size $n$ drawn from (4), is given by

$$
\begin{aligned}
f_{X_{r: n}}(x) & =r\left(\begin{array}{l}
n \\
r
\end{array}\right)(F(x))^{r-1}(1-F(x))^{n-r} f(x) \\
& =r\left(\begin{array}{l}
n \\
r
\end{array}\right) \sum_{k=0}^{r-1}(-1)^{k}\left(\begin{array}{c}
r-1 \\
k
\end{array}\right) \frac{W L D\left(c, \beta(n-r+k+1)^{1 / c}, \theta\right)}{(n-r+k+1)} .
\end{aligned}
$$

From (13), the PDF of the $r$ th order statistic $X_{r: n}$ can be expressed as a finite sum of the Weibull-Lomax PDFs. From (13), the distribution of the sample maximum $X_{n: n}=\max \left(X_{1}, X_{2}, \cdots, X_{n}\right)$, and the sample minimum $X_{1: n}=\min \left(X_{1}, X_{2}, \cdots, X_{n}\right)$ are, respectively, given by

$$
\begin{gathered}
f_{X_{n: n}}(x)=n \sum_{k=0}^{n-1} \frac{(-1)^{k}}{k+1}\left(\begin{array}{c}
n-1 \\
k
\end{array}\right) W L D\left(c, \beta(k+1)^{1 / c}, \theta\right) . \\
f_{X_{1: n}}(x)=W L D\left(c, \beta n^{1 / c}, \theta\right) .
\end{gathered}
$$

From (15), it is evident that the Weibull-Lomax distribution is closed under minimization. This property is also known as min stable property (See Feller, 1971).

In order to find the large sample distribution of $X_{n: n}$, we will use the sufficient condition for weak convergence due to von Mises (1936) which is stated in the following theorem:

Theorem 2.5. Let $F$ be an absolutely continuous c.d.f and suppose $h_{f}(x)$ is nonzero and differentiable function. If

$$
\lim _{x \rightarrow F^{-1}(1)} \frac{d}{d x}\left(\frac{1}{h_{f}(x)}\right)=0,
$$

then $F \in \mathcal{D}\left(G_{1}\right)$, where $G_{1}(x)=\exp (-\exp (x))$.

In our case $F^{-1}(1)=\infty$ and from (6), we have

$$
\lim _{x \rightarrow \infty} \frac{d}{d x}\left(\frac{1}{h_{f}(x)}\right)= \begin{cases}0, & c \geq 1 \\ \infty, & c<1\end{cases}
$$

Hence, the large sample distribution of $X_{n: n}$ is of extreme value type provided that $c>1$. When $c=1$, the Weibull Lomax distribution reduces to the Lomax distribution. Also, according to Arnold et al. (2008), the large sample distribution of $X_{n: n}$ will be of extreme value type.

In order to derive the asymptotic distribution of the sample minima $X_{1: n}$, we consider Theorem 8.3.6 of Arnold et al.(2008). Observe that, since $F^{-1}(0)$ is finite, it follows from the theorem that the asymptotic distribution of the sample minima $X_{1: n}$ is not of Fréchet type. The asymptotic distribution of $X_{1: n}$ will be of the Weibull type with parameter $\alpha>0$ if

$$
\lim _{\varepsilon \rightarrow 0_{+}} \frac{F\left(F^{-1}(0)+\varepsilon x\right)}{F\left(F^{-1}(0)+\varepsilon\right)}=x^{\alpha}, \text { for all } x>0 .
$$

Note that

$$
\lim _{\varepsilon \rightarrow 0_{+}} \frac{F(\theta+\varepsilon x)}{F(\theta+\varepsilon)}=x \lim _{\varepsilon \rightarrow 0_{+}} \frac{f(\theta+\varepsilon x)}{f(\theta+\varepsilon)}=x^{c} .
$$

Hence, the asymptotic distribution of the sample minima $X_{1: n}$ is of the Weibull type with shape parameter $c$. Furthermore, since the WLD is only a shift by $\theta$ of the WPD, therefore, the asymptotic distributions for the sample maxima when $c \geq 1$ is of extreme type. Also, the asymptotic distribution the sample minima for the WPD is of the Weibull type with shape parameter $c$.

Next, we consider the $s$-th moment for $X_{r: n}, 1 \leq r \leq n$. From (13), if $Y \sim W L D\left(c, \beta(n-r+k+1)^{1 / c}, \theta\right)$, then

$$
E\left(Y^{s}\right)=(-1)^{s} \theta^{s}+\theta^{s} \sum_{k=1}^{s} \sum_{i=0}^{\infty}\left(\begin{array}{l}
s \\
k
\end{array}\right) \frac{(-1)^{s-k} k^{i}}{i ! \beta^{i}(n-r+k+1)^{i / c}} \Gamma(1+i / c) .
$$


From (13), the sth moment of $X_{r: n}$ can be written as

$$
E\left(X_{r: n}^{s}\right)=r\left(\begin{array}{l}
n \\
r
\end{array}\right) \sum_{k=0}^{r-1} \frac{(-1)^{k}}{n-r+k+1}\left(\begin{array}{c}
r-1 \\
k
\end{array}\right) E\left(Y^{s}\right)
$$

where $E\left(Y^{s}\right)$ are given in (17). The $s$-th non central moment of $X_{1: n}$ and $X_{n: n}$ can be obtained by setting $r=1$ and $r=n$ in equation (18).

Theorem 2.6. (i) If $c>1$, then the $s$-th non central moment of $X_{r: n}$ exist.

(ii) If $c<1$, then the $s$-th non central moment of $X_{r: n}$ do not exist.

(iii) If $c=1$, then the $s$-th non central moment of $X_{r: n}$ exist iff $\beta(n-r+1)>s$.

Proof. Follows directly from Remark 2.4.

\section{Parameter Estimation and Application}

The parameters of WLD are estimated by using the maximum likelihood. A simulation study is conducted to evaluate the performance of the maximum likelihood method.

\subsection{Maximum Likelihood Estimation Method}

Let $X_{1}, X_{2}, \cdots, X_{n}$ be a random sample of size $n$ drawn from the density in (4). The log-likelihood function $\ell=\ell(c, \beta, \theta)$ is given by

$$
\ell=n c \log \beta+n \log c-\sum_{i=1}^{n} \log \left(\theta+x_{i}\right)+(c-1) \sum_{i=1}^{n} \log \left(\log \left(1+x_{i} / \theta\right)\right)-\sum_{i=1}^{n}\left(\beta \log \left(1+x_{i} / \theta\right)\right)^{c} .
$$

The log-likelihood can be maximized numerically to obtain the maximum likelihood estimates. Several routines available for numerical maximization. We used the PROC NLMIXED in SAS to maximize equation (19). The initial estimates for the parameters of WLD are obtained as follows: the initial estimates of $c$ and $\beta$ are the moment estimates of $c$ and $\beta$ from the Weibull distribution, which are given by $c_{0}=\frac{\pi}{\left(6 s_{\log x_{i}}\right)}$ and $\beta_{0}=\exp \left(-\bar{x}_{\log x_{i}}-\delta / c_{0}\right)$, where $s_{\log x_{i}}$ and $\bar{x}_{\log x_{i}}$ are the sample standard deviation and the sample mean for $\log x_{i}$ and $\delta$ is the Euler gamma constant (Johnson et al., 1994, pp. 642-643). The initial estimate of $\theta$ is taken to be 1 .

\subsection{Simulation Study to Evaluate the Performance of MLE}

In this sub-section, a simulation study is conducted to evaluate the performance of the MLE method of WLD in terms of both bias and standard deviation for various parameter combinations and different sample sizes. For each parameter combination, a random sample from WLD is generated by first generating a random sample $y_{1}, y_{2}, \cdots, y_{n}$ from the Weibull distribution with parameters $c$ and $1 / \beta$, then by using Remark 2.2(i), $X_{i}=\theta\left(\mathrm{e}^{Y_{i}}-1\right), i=1,2, \ldots, n$ follows the $W L D(c, \beta, \theta)$.

The parameter combinations for the simulation study are $c=0.5,1,4,7, \beta=0.5,1,3$, and $\theta=0.5,1,3$. Two different sample sizes of $n=100$ and 500 are used in the simulation. For each sample size and parameter combination, the simulation process is repeated 200 times. The average bias (estimate - actual), and the average standard deviation of the maximum likelihood estimates are presented in Tables 1 and 2.

The results show that the maximum likelihood estimation method performs well. In general, the biases and standard deviations of the parameters are reasonably small. As the sample size increases, the results show that the biases and standard deviations of the estimators decrease. The results from this simulation study, suggest that the maximum likelihood estimates method can be used effectively to estimate the parameters of the Weibull-Lomax distribution. Also, if $c>1$, the results from Tables 1 and 2 show that the MLE method performs good in estimating the model parameters. This suggests that the WLD can be used an alternative to the WPD in modeling real life data sets.

\subsection{Applications}

Alzaatreh et al. (2013a) used three data sets from Park et al. (1964) and Park (1954) and fit them to Weibull-Pareto distribution using the MMLE method. In this subsection, we fit these data sets and show that WLD provides similar fit. The data sets are the grouped frequency distributions of adult numbers for Tribolium Confusum and Tribolium Castaneum cultured at $24 \mathrm{C}$ and Tribolium Confusum strain. In particular, Data set 1 represents a random sample of 857 Tribolium 
Table 1. Bias and standard deviation of the parameter estimates for $n=100$

\begin{tabular}{|c|c|c|c|c|c|c|c|c|}
\hline \multicolumn{3}{|c|}{ Actual Values } & \multicolumn{3}{|c|}{ Bias } & \multicolumn{3}{|c|}{ Standard deviation } \\
\hline$c$ & $\beta$ & $\theta$ & $\hat{c}$ & $\hat{\beta}$ & $\hat{\theta}$ & $\hat{\hat{c}}$ & $\hat{\beta}$ & $\hat{\theta}$ \\
\hline \multirow[t]{9}{*}{0.5} & 0.5 & 0.5 & 0.0287 & -0.0001 & 0.0218 & 0.0748 & 0.1764 & 0.4158 \\
\hline & & 1 & 0.0225 & 0.0073 & 0.1774 & 0.0784 & 0.1920 & 0.9007 \\
\hline & & 3 & 0.0266 & 0.0092 & 0.3276 & 0.0772 & 0.1826 & 2.5553 \\
\hline & 1 & 0.5 & 0.0232 & -0.0011 & 0.0184 & 0.0683 & 0.3520 & 0.3326 \\
\hline & & 1 & 0.0218 & 0.0086 & 0.1598 & 0.0681 & 0.3814 & 0.9051 \\
\hline & & 3 & 0.0223 & 0.0165 & 0.3531 & 0.0626 & 0.3643 & 2.1206 \\
\hline & 3 & 0.5 & 0.0206 & 0.0176 & 0.0258 & 0.0517 & 1.1344 & 0.2790 \\
\hline & & 1 & 0.0241 & 0.1880 & 0.1011 & 0.0497 & 1.1983 & 0.6267 \\
\hline & & 3 & 0.0154 & 0.1619 & 0.1682 & 0.0478 & 1.1155 & 1.6073 \\
\hline \multirow[t]{9}{*}{1} & 0.5 & 0.5 & 0.0836 & 0.0083 & 0.0680 & 0.2328 & 0.1924 & 0.4886 \\
\hline & & 1 & 0.0500 & 0.0227 & 0.2613 & 0.2244 & 0.1856 & 0.9557 \\
\hline & & 3 & 0.0773 & 0.0168 & 0.5588 & 0.2333 & 0.1861 & 2.9163 \\
\hline & 1 & 0.5 & 0.0333 & 0.0658 & 0.0743 & 0.1542 & 0.3902 & 0.3491 \\
\hline & & 1 & 0.0472 & 0.0258 & 0.0940 & 0.1596 & 0.3618 & 0.6508 \\
\hline & & 3 & 0.0556 & 0.0495 & 0.3845 & 0.1691 & 0.3917 & 2.1340 \\
\hline & 3 & 0.5 & 0.0408 & 0.0797 & 0.0193 & 0.1052 & 1.1522 & 0.2372 \\
\hline & & 1 & 0.0376 & 0.0614 & 0.0085 & 0.1022 & 1.1650 & 0.4817 \\
\hline & & 3 & 0.0256 & 0.1354 & 0.1569 & 0.0955 & 1.1701 & 1.4879 \\
\hline \multirow[t]{9}{*}{4} & 0.5 & 0.5 & 0.5229 & -0.0268 & -0.0256 & 1.0086 & 0.1698 & 0.4036 \\
\hline & & 1 & 0.3374 & -0.0004 & 0.0775 & 0.8892 & 0.1671 & 0.8325 \\
\hline & & 3 & 0.6047 & -0.0413 & -0.3706 & 1.0022 & 0.1418 & 1.9807 \\
\hline & 1 & 0.5 & 0.6234 & -0.0505 & -0.0246 & 0.9791 & 0.4286 & 0.3377 \\
\hline & & 1 & 0.4174 & 0.0260 & 0.0676 & 0.9472 & 0.4471 & 0.7082 \\
\hline & & 3 & 0.4091 & 0.0246 & 0.1803 & 1.0248 & 0.4609 & 2.1711 \\
\hline & 3 & 0.5 & 0.1886 & -0.1328 & -0.0265 & 0.4220 & 1.1250 & 0.2178 \\
\hline & & 1 & 0.1209 & 0.1592 & 0.0614 & 0.4010 & 1.2174 & 0.4728 \\
\hline & & 3 & 0.1377 & -0.0921 & -0.1133 & 0.4029 & 1.1831 & 1.3794 \\
\hline \multirow[t]{9}{*}{7} & 0.5 & 0.5 & 0.4476 & -0.0086 & 0.0007 & 1.3689 & 0.1239 & 0.2988 \\
\hline & & 1 & 0.4629 & -0.0004 & 0.0396 & 1.3946 & 0.1250 & 0.6038 \\
\hline & & 3 & 0.6692 & -0.0191 & -0.1366 & 1.5165 & 0.1097 & 1.5466 \\
\hline & 1 & 0.5 & 1.8592 & -0.1978 & -0.1348 & 1.7858 & 0.4377 & 0.3463 \\
\hline & & 1 & 1.6115 & -0.1439 & -0.1945 & 1.9261 & 0.4591 & 0.7135 \\
\hline & & 3 & 1.9645 & -0.2476 & -1.0595 & 1.7831 & 0.4022 & 1.8825 \\
\hline & 3 & 0.5 & 0.2483 & 0.0982 & 0.0197 & 0.7125 & 1.1624 & 0.2273 \\
\hline & & 1 & 0.2000 & 0.0234 & 0.0083 & 0.6995 & 1.2274 & 0.4811 \\
\hline & & 3 & 0.2757 & -0.0104 & -0.0079 & 0.6927 & 1.2099 & 1.4260 \\
\hline
\end{tabular}


Table 2. Bias and standard deviation of the parameter estimates for $n=500$

\begin{tabular}{|c|c|c|c|c|c|c|c|c|}
\hline \multicolumn{3}{|c|}{ Actual Values } & \multicolumn{3}{|c|}{ Bias } & \multicolumn{3}{|c|}{ Standard deviation } \\
\hline$c$ & $\beta$ & $\theta$ & $\hat{c}$ & $\hat{\beta}$ & $\hat{\theta}$ & $\hat{c}$ & $\hat{\beta}$ & $\hat{\theta}$ \\
\hline \multirow[t]{9}{*}{0.5} & 0.5 & 0.5 & 0.0132 & -0.0151 & -0.0281 & 0.0348 & 0.0944 & 0.1861 \\
\hline & & 1 & 0.0116 & -0.0030 & -0.0058 & 0.0341 & 0.0974 & 0.3857 \\
\hline & & 3 & 0.0067 & 0.0026 & 0.0893 & 0.0372 & 0.0978 & 1.2534 \\
\hline & 1 & 0.5 & 0.0079 & 0.0138 & 0.0189 & 0.0356 & 0.2224 & 0.2066 \\
\hline & & 1 & 0.0077 & 0.0057 & 0.0303 & 0.0359 & 0.2250 & 0.4078 \\
\hline & & 3 & 0.0138 & -0.0107 & 0.0092 & 0.0384 & 0.2345 & 1.1882 \\
\hline & 3 & 0.5 & 0.0057 & 0.1076 & 0.0213 & 0.0298 & 0.9001 & 0.1922 \\
\hline & & 1 & 0.0073 & 0.0660 & 0.0442 & 0.0321 & 0.8795 & 0.4162 \\
\hline & & 3 & 0.0060 & 0.0491 & 0.0766 & 0.0302 & 0.8460 & 1.1589 \\
\hline \multirow[t]{9}{*}{1} & 0.5 & 0.5 & 0.0098 & 0.0221 & 0.0816 & 0.1273 & 0.1266 & 0.3280 \\
\hline & & 1 & 0.0171 & 0.0143 & 0.1173 & 0.1333 & 0.1168 & 0.5645 \\
\hline & & 3 & 0.0253 & 0.0084 & 0.2387 & 0.1228 & 0.1140 & 1.7001 \\
\hline & 1 & 0.5 & 0.0117 & 0.0198 & 0.0211 & 0.0962 & 0.2375 & 0.2007 \\
\hline & & 1 & 0.0200 & 0.0162 & 0.0540 & 0.1017 & 0.2642 & 0.4483 \\
\hline & & 3 & 0.0161 & 0.0260 & 0.1870 & 0.0987 & 0.2624 & 1.3323 \\
\hline & 3 & 0.5 & 0.0179 & 0.1027 & 0.0238 & 0.0676 & 1.0732 & 0.2246 \\
\hline & & 1 & 0.0118 & 0.1047 & 0.0476 & 0.0602 & 0.9458 & 0.3995 \\
\hline & & 3 & 0.0112 & 0.1673 & 0.2464 & 0.0672 & 1.0295 & 1.3051 \\
\hline \multirow[t]{9}{*}{4} & 0.5 & 0.5 & 0.1038 & 0.0497 & 0.1590 & 0.9285 & 0.1857 & 0.4613 \\
\hline & & 1 & 0.0944 & 0.0491 & 0.3146 & 0.9194 & 0.1861 & 0.9304 \\
\hline & & 3 & 0.3544 & -0.0079 & 0.0952 & 0.9766 & 0.1537 & 2.1699 \\
\hline & 1 & 0.5 & 0.0156 & 0.1598 & 0.1371 & 0.6922 & 0.3962 & 0.3200 \\
\hline & & 1 & 0.0250 & 0.1420 & 0.2442 & 0.6449 & 0.3926 & 0.6303 \\
\hline & & 3 & -0.0064 & 0.1548 & 0.7849 & 0.6285 & 0.3846 & 1.8555 \\
\hline & 3 & 0.5 & 0.0956 & 0.0416 & 0.0097 & 0.2913 & 1.2129 & 0.2396 \\
\hline & & 1 & 0.0868 & 0.0659 & 0.0288 & 0.2875 & 1.2269 & 0.4841 \\
\hline & & 3 & 0.1223 & 0.0032 & 0.0094 & 0.3002 & 1.2342 & 1.4565 \\
\hline \multirow[t]{9}{*}{7} & 0.5 & 0.5 & 0.2962 & 0.0415 & 0.1375 & 1.2609 & 0.1857 & 0.4586 \\
\hline & & 1 & -0.0383 & 0.0600 & 0.3516 & 1.1002 & 0.1743 & 0.8852 \\
\hline & & 3 & -0.0157 & 0.0398 & 0.7004 & 1.1850 & 0.1412 & 2.1105 \\
\hline & 1 & 0.5 & 0.5314 & 0.0559 & 0.0571 & 1.5608 & 0.4655 & 0.3683 \\
\hline & & 1 & 0.5067 & 0.0802 & 0.1527 & 1.5718 & 0.4696 & 0.7455 \\
\hline & & 3 & 0.5462 & 0.0624 & 0.3714 & 1.6205 & 0.4495 & 2.1272 \\
\hline & 3 & 0.5 & 0.3919 & -0.4290 & -0.0834 & 0.5326 & 1.1271 & 0.2209 \\
\hline & & 1 & 0.0085 & 0.2958 & 0.1175 & 0.4168 & 1.0852 & 0.4266 \\
\hline & & 3 & 0.1736 & 0.0318 & 0.0439 & 0.5122 & 1.2105 & 1.4283 \\
\hline
\end{tabular}


Table 3. Parameter estimates and K-S values for the fitted data sets

\begin{tabular}{|c|cc|cc|cc|}
\hline Data sets & \multicolumn{2}{|c|}{ Data set 1 } & \multicolumn{2}{c|}{ Data set 2 } & \multicolumn{2}{c|}{ Data set 3 } \\
\hline Distributions & $W P D$ & $W L D$ & $W P D$ & $W L D$ & $W P D$ & $W L D$ \\
\hline Estimates & $\hat{\theta}=9.107$ & $\hat{\theta}=14.245$ & $\hat{\theta}=33.208$ & $\hat{\theta}=22.122$ & $\hat{\theta}=23.283$ & $\hat{\theta}=12.627$ \\
& $\hat{\beta}=0.380$ & $\hat{\beta}=0.436$ & $\hat{\beta}=0.747$ & $\hat{\beta}=0.521$ & $\hat{\beta}=0.866$ & $\hat{\beta}=0.517$ \\
& $\hat{c}=6.953$ & $\hat{c}=6.863$ & $\hat{c}=5.059$ & $\hat{c}=8.719$ & $\hat{c}=6.161$ & $\hat{c}=12.398$ \\
\hline K-S values & 0.0482 & 0.0549 & 0.0730 & 0.0905 & 0.0803 & 0.0901 \\
AIC values & 8904.5 & 8908.6 & 9276.41 & 9311.5 & 2973.44 & 2982.1 \\
\hline
\end{tabular}

Castaneum cultured at $24^{\circ} \mathrm{C}$, the Data set 2 represents a random sample of 952 Tribolium Castaneum cultured at $24^{0} \mathrm{C}$ and Data set 3 represents a random sample of 368 Tribolium Castaneum cultured at $24^{0} C$. The data sets are avialable in Alzaatreh, et al. (2013a). Above we provide the estimates, the Kolmogorov-Smirnov test (K-S) and the Akaike information criterion (AIC) values for the WLD and WPD in Table 3. The estimates of WLD are based on MLE method and the estimates of WPD are based on MMLE method (taken from Alzaatreh et al., 2013a). The results in Table 3 show that WLD and WPD provide similar fits to three data sets. Figure 5 supports the results in Table 3.
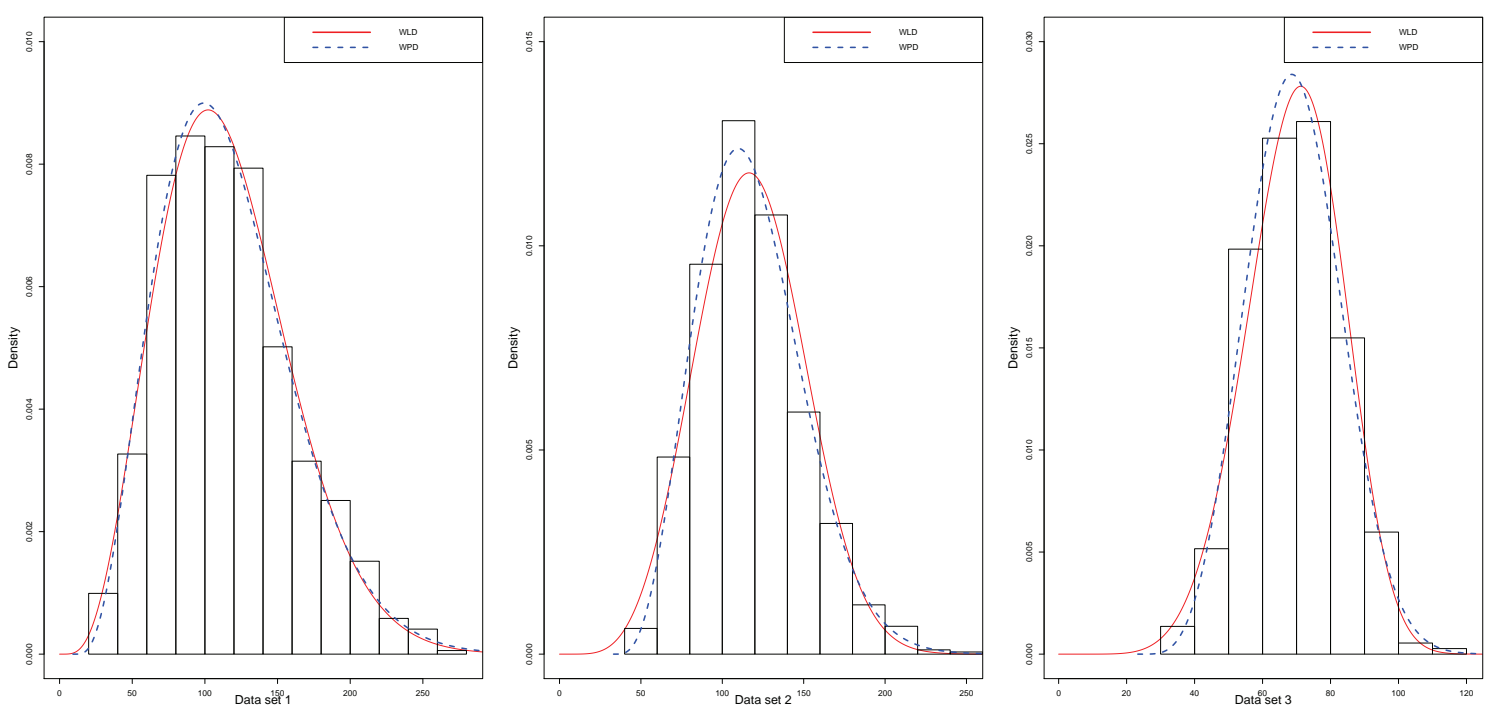

Figure 5. Histograms and fitted PDFs for WLD and WPD.

\section{Conclusion}

In this paper, a shift by a parameter $\theta>0$ of the Weibull-Pareto distribution defined by Alzaatreh et al. (2013a) is proposed namely, the Weibull-Lomax distribution. Based on Alzaatreh et al. (2013a), the maximum likelihood estimation produces high biases and standard errors for the WPD parameters and therefore, they proposed a modification of the MLE, MMLE, which can be used only when $c>1$. This problem in estimatioing the WPD parameters has motivated us to present an alternative to the WPD by using Lomax distribution in replace of Pareto distribution in the $T-X$ family proposed by Alzaatreh et al. (2013b). In this paper we showed that the MLE method can be used effectively to estimate the WLD parameters without any restriction on the parameter $c$. The results of the simulation study in section 3.2 are compared with the results of the simulation study for the WPD obtained by Alzaatreh et al. (2013a). In most cases, it was observed that the biases and standard errors for the MLEs of WLD parameters are smaller than the MMLEs for the WPD parameters. Furthermore, WLD is applied to the same data sets used in Alzaatreh et al. (2013a). The results in Table 3 and Figure 5 showed that WLD and WPD provide similar fit to the data sets.

\section{Acknowledgments}

The authors are grateful for the comments and suggestions by the referees and the Editor-in-Chief. Their comments and suggestions have greatly improved the paper. 


\section{References}

Alzaatreh, A., Famoye, F. and Lee, C. (2013a). Weibull-Pareto distribution and its applications, Communications in Statistics:Theory and Methods, 42, 1673-1691. http://dx.doi.org/10.1080/03610926.2011.599002.

Alzaatreh, A. and Ghosh, I. (2014). On the Weibull-X family of distributions, Journal of Statistical Theory and Applications, 14, 169-183.

Alzaatreh, A., Lee, C. and Famoye, F. (2013b). A new method for generating families of continuous distributions, Metron, 71, 63-79. http://dx.doi.org/10.1007/s40300-013-0007-y.

Arnold, B.C., Balakrishnan, N. and Nagarajah, H.N. (2008). A first course in Order Statistics, John Wiley, New York. http://dx.doi.org/10.1137/1.9780898719062 .

Feller, W. (1971). An introduction to probability theory and its applications, John Wiley, N.Y.

Galton, F. (1883). Enquiries into Human Faculty and its Development, Macmillan and Company, London.

Hall, I.J. (1984). Approximate one-sided tolerance limits for the difference or sum of two independent normal variates, Journal of Qualitative Technology, 16, 15-19.

Johnson, N. L., Kotz, S., Balakrishnan, N. (1994). Continuous Univariate Distributions, 2nd ed. Vol. 1. New York: John Wiley \& Sons.

Moors, J.J. (1988). A quantile alternative for kurtosis, Statistician, 37, 25C32. http://dx.doi.org/10.2307/2348376 .

Park, T. (1954). Experimental studies of interspecies competition II. Temperature, humidity, and competition in two species of Tribolium, Physiolog. Zool., 27, 177-238. http://dx.doi.org/10.1086/physzool.27.3.30152164.

Park, T., Leslie, P. H, Mertz, D. B. (1964). Genetic strains and competition in population of Tribolium, Physiolog. Zool., 37, 97-162. http://dx.doi.org/10.1086/physzool.37.2.30152328.

Shannon, C. E. (1948). A mathematical theory of communication, Bell Syst. Tech. J., 27, 379-432.

von Mises, R. (1936). La distribution de la plus grande de n valeurs, Review of Mathematics Union Interbalcanique, 1 , 141-160.

Weerahandi, S. and Johnson, R.A. (1992). Testing reliability in a stress-strength model when $X$ and $Y$ are normally distributed, Technometrics, 38, 83-91. http://dx.doi.org/10.2307/1269555.

Appendix R outputs for the regression line in Figure 4.

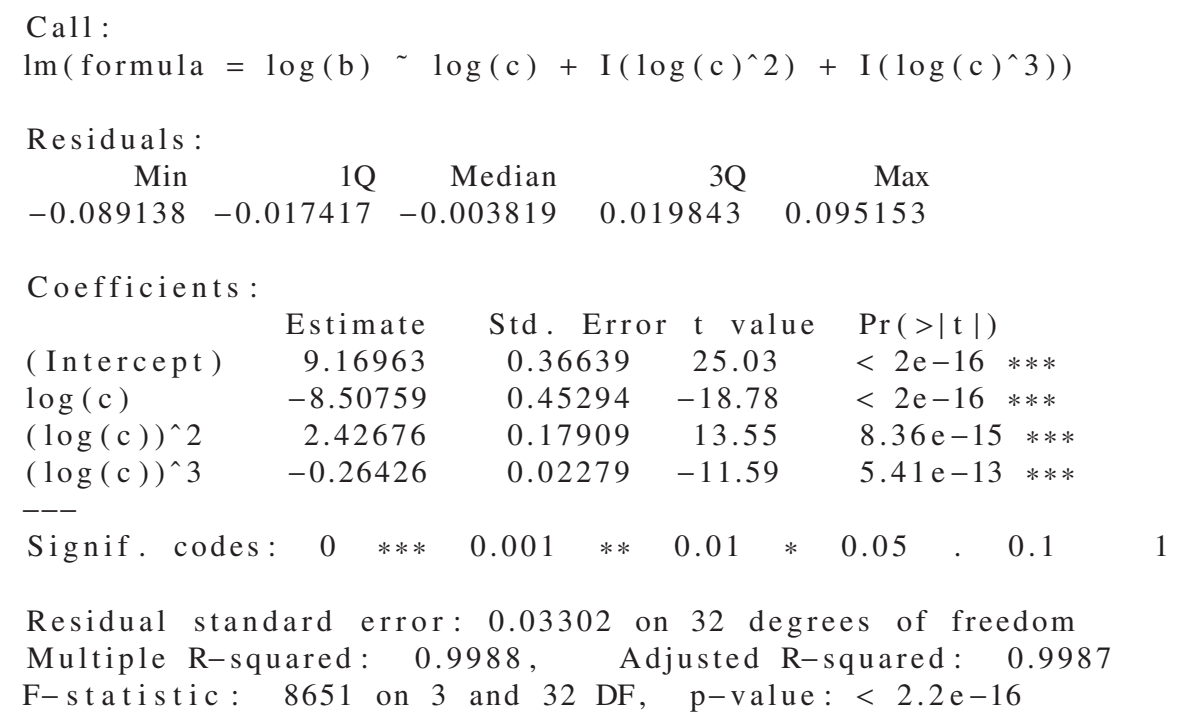

\section{Copyrights}

Copyright for this article is retained by the author(s), with first publication rights granted to the journal.

This is an open-access article distributed under the terms and conditions of the Creative Commons Attribution license (http://creativecommons.org/licenses/by/3.0/). 